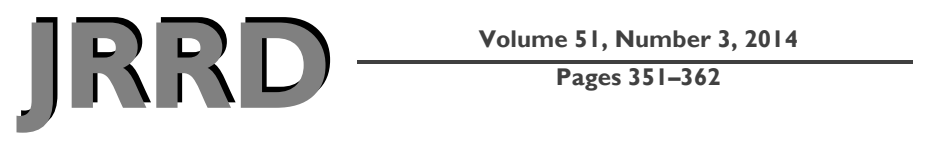

\title{
Self-reported and performance-based outcomes using DEKA Arm
}

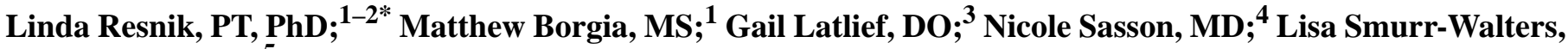 \\ MS, OTR/L, CHT \\ ${ }^{1}$ Providence Department of Veterans Affairs (VA) Medical Center, Providence, RI; ${ }^{2}$ Department of Health Services, Policy, \\ and Practice, Brown University, Providence, RI; ${ }^{3}$ James A. Haley Veterans' Hospital, Tampa, FL; ${ }^{4}$ VA New York Harbor \\ Healthcare System, New York, NY; ${ }^{5}$ Center for the Intrepid, Brooke Army Medical Center, Fort Sam Houston, TX
}

\begin{abstract}
Mechanical properties of the DEKA Arm and associated engineering innovations are easy to observe. What is less clear is how these advances translate into functional benefits for the user with amputation. Study aims were to (1) quantify outcomes including dexterity, performance of daily activities, and prosthetic skill and spontaneity of users of the DEKA Arm and (2) compare outcomes when using the DEKA Arm with scores using the existing prosthesis. This was a quasi-experimental study. Descriptive analyses examined outcomes by DEKA Arm configuration level. Of the 39 subjects fit with a DEKA Arm, 32 were trained in use and completed end-of-study testing. Data from 26 prosthetic users were used to compare outcomes using existing prostheses with outcomes with the DEKA Arm. Dexterity and activity performance with the DEKA Arm varied by amputation level $(p<0.01)$. Self-reported function and number of activities performed using the prosthesis were similar across levels. Comparisons with existing prostheses showed the effect on dexterity varied by level. Activity performance and spontaneity of prosthetic use improved for users of the shoulder configuration level, while use of the prosthesis to perform activities and perceived difficulty performing self-selected tasks improved for all levels.
\end{abstract}

Key words: amputation, assistive technology, Department of Veterans Affairs, dexterity, disability evaluation, outcomes, physical function, prosthetics, rehabilitation, upper limb.

\section{INTRODUCTION}

Persons with upper-limb amputation are not satisfied with the current state of technology, and many do not use a prosthesis [1-2]. Reasons for rejection include negative initial prosthetic experience due to poor socket fit and discomfort, weight of the device, and/or late fitting; dissatisfaction with device functionality, lack of tactile sensation, and appearance; repeated mechanical failure; and costliness of repairs [2-9]. However, the level of amputation is

\footnotetext{
Abbreviations: AM-ULA = Activities Measure for Upper-Limb Amputees, BAMC $=$ Brooke Army Medical Center, BB $=$ Modified Box and Block Test of Manual Dexterity, DARPA = Defense Advanced Research Projects Agency, EMG = electromyography, gen 2 = second-generation DEKA Arm, gen 3 = third-generation DEKA Arm, HC = humeral configuration, JTHF = Jebsen-Taylor Hand Function Test, NYHHS = New York Harbor Healthcare System, OT = occupational therapist, PSFS = Patient-Specific Functional Scale, RC = radial configuration, RR\&D = Rehabilitation Research and Development, SC = shoulder configuration, $\mathrm{SD}=$ shoulder disarticulation, $\mathrm{TH}=$ transhumeral, $\mathrm{TR}=$ transradial, UEFS = Upper-Extremity Functional Scale, UNB = University of New Brunswick Test of Prosthetic Function for Unilateral Amputees, VA = Department of Veterans Affairs.

*Address all correspondence to Linda Resnik, PT, PhD; Providence VA Medical Center, 830 Chalkstone Ave, Providence, RI 02908; 401-273-7100, ext 2368.

Email: Linda.Resnik@va.gov

http://dx.doi.org/10.1682/JRRD.2013.08.0180
} 
the most significant factor in upper-limb prosthesis rejection [1,10-11]. The rate of rejection for persons with shoulder disarticulation (SD) is 60 percent, the rate for transhumeral $(\mathrm{TH})$ users is 57 percent, and the rate for transradial (TR) users is 6 percent.

Persons with upper-limb amputation currently have the choice of three basic types of prosthetic devices: body-powered devices controlled by a cable activated through a shoulder harness, motorized devices controlled with electromyography (EMG) signals from the residual limb (also known as myoelectric prostheses), or hybrid devices that use a combination of body-powered and EMG controls. Most devices currently on the market offer terminal devices that open and close in a single-grip pattern. Several newer myoelectric hand designs enable the user to select from multiple grips with prepositioning of the thumb. Myoelectric devices can also offer powered wrist pronation and supination and powered elbow flexion and extension. However, users with more than one type of powered movement are only able to control one movement at a time. There are currently no powered prosthetic shoulders on the commercial market. Those with proximal limb loss may use fixed shoulder joints or manually locking shoulder joints that allow the user to preposition the limb for specific tasks.

The DEKA Arm [12], a precommercial upper-limb prosthetic prototype funded by the Defense Advanced Research Projects Agency (DARPA) Revolutionizing Prosthetics Program [13], was engineered to provide the potential for significantly greater upper-limb function than devices currently on the market. The full DEKA Arm allows up to $10^{\circ}$ of powered movement and six preprogrammed hand grips. The powered elbow provides flexion and extension as well as humeral rotation. The powered wrist flexes and extends as well as pronates and supinates and provides a combined lateral and medial deviation capability. The device is operated by a combination of methods that includes foot controls, optional EMG controls, pressure switches, or other commonly available prosthetic input elements. The DEKA Arm system that includes a powered shoulder employs end-point control that enables simultaneous control of multiple arm joints, minimizing the cognitive burden required to control all upper-limb joints separately [14-15]. The mechanical properties of the DEKA Arm are easy to observe. It is less clear how these advances translate into real-world, functional benefits for the user with amputation.
In 2008, the Department of Veterans Affairs (VA) Rehabilitation Research and Development (RR\&D) Service funded an optimization study. One of the major purposes of this usability study [16] was to examine the ease of use, users' perceptions about the device attributes, and desirability of the device. DEKA used feedback from the VA study and from studies of their own subjects to refine the second-generation DEKA Arm (gen 2) prototype and design the third-generation DEKA Arm (gen 3) prototype. During the usability study, the VA also performed extensive testing to quantify what activities subjects were able to do with their existing prostheses and what they were able to achieve when using the gen 2 and gen 3 prototypes. Analyses of qualitative study data found that the majority of subjects wanted to receive a DEKA Arm, largely because of the new activities that they could perform using the DEKA Arm [17]. The purposes of this article are to report on quantitative study findings, specifically to (1) quantify outcomes, including dexterity, performance of daily activities, and prosthetic skill and spontaneity of users of the DEKA Arm and (2) compare outcomes scores when using the DEKA Arm with scores using the existing prosthesis.

\section{METHODS}

This was a quasi-experimental study with repeated measures, prior to and after training with the DEKA Arm. This study was conducted as part of the overall VA study to optimize the DEKA Arm. Participating sites included the Providence VA Medical Center; VA New York Harbor Healthcare System (NYHHS); James A. Haley Veterans' Hospital; VA Long Beach Healthcare System; and Center for the Intrepid, Brooke Army Medical Center (BAMC). The study was approved by each site's institutional review board. Each site was staffed by a study physician, research assistant, and one or more prosthetists and occupational therapists (OTs).

Subjects were recruited through clinical staff, advertisements, and press releases. Subjects were purposefully sampled to include participants (both prosthetic users and nonusers) at three configuration levels who would be trained to use the DEKA Arm: radial configuration (RC), humeral configuration (HC), and shoulder configuration (SC). Subjects met the following inclusion criteria: at least $18 \mathrm{yr}$ old and single or bilateral upper-limb amputation at the TR, TH, SD, or scapula-thoracic level. All 
subjects were required to have active control over one or both ankles or have an appropriate number of myoelectric and/or other control sites to enable adequate prosthetic controls configuration for the DEKA Arm (as determined by the study prosthetist). Subjects were excluded if they had significant uncorrectable visual deficits; major communication or neurocognitive deficits; skin conditions that prevented prosthetic wear; an electrically controlled medical device; or any significant comorbidity, cognitive deficit, or mental health problem that would limit their ability to participate fully. Subjects were enrolled based on their availability to participate, the availability of a DEKA Arm at the necessary configuration level, and our desire to balance the sample in terms of configuration level and sex. In keeping with VA policy, whenever possible, Veterans and Active Duty persons were prioritized in the enrollment.

After socket fitting and setup of DEKA Arm controls, subjects were oriented to the device features and controls through an interactive virtual reality environment software program [14] and then trained in the use of the device by the study therapists using a standardized protocol. Prosthetic use training began with reinforcement of prosthetic control patterns of motions, proceeded to simple grasp and release activities, and progressed to more complex functional tasks. For most subjects, training took place over the course of ten $2 \mathrm{~h}$ sessions. Partway through the study, the training protocol was adapted for SC subjects by adding five additional training visits to allow this group of participants more time to master the more complex SC device. Of the 14 people using an SC, 8 completed 15 training visits.

\section{Outcome Measures}

Outcome measures were administered at the first study visit (visit 1) and again 1 wk later (visit 2) using the subject's existing prosthesis, readministered at the onset of training with the DEKA Arm, and after every five training visits until the end of the study. Outcome measures of activity performance included four performancebased measures: the Modified Box and Block Test of Manual Dexterity (BB) [18-20], the Jebsen-Taylor Hand Function Test (JTHF) [21], the Activities Measure for Upper-Limb Amputees (AM-ULA) [22], the University of New Brunswick Test of Prosthetic Function for Unilateral Amputees (UNB) [23]; and two self-report measures: the Upper-Extremity Functional Scale (UEFS) from the Orthotics and Prosthetics Users Survey [24-25] and the
Patient-Specific Functional Scale (PSFS) [26]. All performance-based measures were administered by OTs.

The BB consists of a box with a center partition [1820]. Small wooden blocks were placed in one side of the box, and the subject was asked to use the prosthetic terminal device to grasp one block at a time, transport it over the partition, and release it. The number of blocks transported to the other side in $60 \mathrm{~s}$ was counted. It has excellent reliability and known group validity for upperlimb amputation [27].

The JTHF is a seven-part dexterity test that evaluates the time needed to perform seven tasks, including (1) writing a 24-letter, third-grade reading difficulty sentence; (2) turning over $7.6 \times 12.7 \mathrm{~cm}(3 \times 5 \mathrm{in}$.) cards in simulated page turning; (3) picking up small common objects (including pennies, paper clips, bottle caps) and placing them in a container; (4) stacking checkers; (5) simulated feeding; (6) moving large empty cans; and (7) moving large $1 \mathrm{lb}$ cans [21]. Test administration and scoring were modified by capping the maximal allowable time for each subtask at 2 min. Rather than calculating the time to complete all items in the subtask, the number of items completed per second was calculated. This administration method has acceptable reliability and known group validity [27].

The AM-ULA is an 18-item measure that assesses key elements of functional performance with a prosthesis: the ability of the participant to complete daily activities, speed of the performance, movement quality, skillfulness of prosthetic use, and independence [22]. AM-ULA task performance was videotaped and graded by a blinded, certified hand therapist rater who was trained in the test scoring criteria [22]. Because this measure was developed during this study, it was administered to most, but not all, subjects. Our analysis of psychometric properties showed that it had excellent test-retest reliability, interrater reliability, and internal consistency and demonstrated known group validity [22]. Higher AMULA scores indicate better performance.

The subtest of the UNB that we used was designed for 11 to 13 yr olds [23]. This subtest included 10 activities related to (1) wrapping a parcel, (2) sewing a button on cloth, (3) cutting meat, (4) drying dishes, and (5) sweeping floors. Each activity is rated with a dual rating scale on spontaneity of prosthetic use (Spontaneity) and skill of prosthetic use (Skill). UNB task performance was videotaped and graded by a blinded, certified hand therapist rater who was trained in the test scoring criteria [28]. Our 
analyses of the psychometrics of the UNB found that the subtest had acceptable internal consistency, test-retest, and interrater reliability and preliminary evidence of validity [28]. Higher UNB scores indicate better performance.

The UEFS items ask participants to evaluate the ease of performing 23 activities, including self-care and instrumental daily living tasks, using a 5-point scale from 1 (very easy) to 5 (cannot perform). Items include activities varying from face washing, buttoning a shirt, tying shoelaces, using a fork or spoon, and name writing to donning and doffing the prosthesis [24-25]. In our study, we used 22 of the 23 UEFS items, omitting the one item related to washing. Because of this, we used item response theory methods in WINSTEPS [29] to recalibrate the measure scores and calculate person-level summary scores (UEFS summary). Reliability of the modified UEFS was 0.80 [22]. The UEFS also asks respondents to indicate whether or not they usually perform each of the activities using or not using their prosthesis (or orthosis). The UEFS use scale was scored by calculating the proportion of activities that the subject indicated that they performed using the prosthesis.

The PSFS asks subjects to identify up to five activities that they have difficulty performing due to their condition and then rate the amount of limitation they have in performing these activities on a scale of 0 to 10 , with 0 being unable to perform the activity and 10 being able to perform the activity with no problem. Individual items are scored separately. The PSFS has been shown to be valid and responsive to change for patients with arm impairments [30], neck pain, cervical radiculopathy, knee pain, and low back pain [31-32]. Our earlier analyses provided evidence of its validity for participants with upper-limb amputation [22].

\section{Data Analysis}

Descriptive statistics (means, ranges, standard deviations) of demographic data for subjects who participated in gen 2 , gen 3 , and only screening activities were examined. Descriptive statistics for all quantitative measures at baseline (with existing prosthesis) and at the end of the study period (after training with the DEKA Arm) were examined. Data from visits 1 and 2 were used to calculate test-retest reliability of measures (reported elsewhere) [22,27-28]. End-of-study outcome data were compared by level of prosthesis used (RC, HC, and SC) using the Kruskal-Wallis one-way analysis of variance by ranks test. Results for gen 2 and gen 3 subsamples were com- pared graphically. Outcomes using the existing prosthesis were compared with those using the DEKA Arm at the end of the study using two-sided paired $t$-tests. Because of the small sample size, these analyses were repeated using nonparametric Wilcoxon signed-rank tests. To determine whether the effect of the DEKA Arm varied by amputation level, the data were stratified by DEKA Arm configuration level and the analysis repeated using the Wilcoxon signed-rank test.

\section{RESULTS}

Of the 75 subjects screened, 39 were fit with a DEKA Arm; 26 were fit under the gen 2 protocol (1 participated in the pilot and then repeated participation in the gen 2 protocol) and 13 under the gen 3 protocol (Table 1). Of the subjects, 12 were fit with an RC, 13 with an HC, and 14 with an SC. The Figure shows the flow of subjects through the study. Seven subjects terminated the study after eight or fewer training visits. Thirty-one subjects completed all specified training visits and one subject completed 9 of 10 training visits before end-of-study testing. These 32 participants comprise the completers group.

Table 2 shows the results of Kruskal-Wallis tests at the end of the study by DEKA Arm configuration level. Scores of dexterity and activity performance measures were consistently better for RC users than HC and SC users. There were no significant differences in self-report of activity performance or in UNB skill or spontaneity scores by DEKA Arm configuration level. Table 3 shows mean outcome scores for completers using the gen 2 prototype compared with completers using the gen 3 prototype by level of fit. No clear pattern of superiority of one prototype over the other was observed.

Comparison of existing device with the DEKA Arm was made for 26 study completers who were prosthetic users at baseline (Table 4). To enhance internal validity, 3 of the 29 subjects who were categorized as prosthetic users at baseline were not included in this comparison; one because he was fit at the SC level but had an averagelength humeral residuum (he had been fit with an SC so that we could obtain usability feedback on this configuration level), one because he was a very new prosthetic user (1 mo) at baseline testing, and one because he used a cosmetic prosthesis only.

As measured by the BB and JTHF feeding test, dexterity was significantly slower with the DEKA Arm than 
Table 1.

Characteristics of all screened subjects.

\begin{tabular}{|c|c|c|c|}
\hline Characteristic & Gen $2(n=26)$ & Gen $3(n=13)$ & $\begin{array}{l}\text { Screened But Not Enrolled } \\
\qquad(n=36)\end{array}$ \\
\hline Age, yr (mean \pm SD [range]) & $45.4 \pm 16.7(19.7-82.8)$ & $46.4 \pm 16.4(23.1-70.8)$ & $43.1 \pm 14.6(19.9-79.4)$ \\
\hline \multicolumn{4}{|l|}{ DEKA Arm Configuration Level, $n$} \\
\hline Radial & 8 & 4 & NA \\
\hline Humeral & 8 & 5 & NA \\
\hline Shoulder & 10 & 4 & NA \\
\hline Female & $4(15.4)$ & $1(7.7)$ & $3(8.3)$ \\
\hline \multicolumn{4}{|l|}{ Race, $n(\%)$} \\
\hline White & $23(88.5)$ & $13(100.0)$ & $29(80.6)$ \\
\hline Other & $3(11.5)$ & $0(0.0)$ & 7 (19.4) \\
\hline \multicolumn{4}{|l|}{ Military Status, $n(\%)$} \\
\hline \multicolumn{4}{|c|}{ Prosthetic User (active device only), $n$ (\%) } \\
\hline Not Current User & $3(11.5)$ & $2(15.4)$ & $7(19.4)$ \\
\hline Full-Time & $14(53.9)$ & $6(46.2)$ & $21(58.3)$ \\
\hline Part-Time & $9(34.6)$ & 5 (38.5) & $8(22.2)$ \\
\hline Participant in Gen 2 Study, $n$ (\%) & $26(100.0)$ & $5(38.5)$ & NA \\
\hline \multicolumn{4}{|c|}{ Prosthetic Experience (includes cosmetic), $n$ (\%) } \\
\hline Very New (<3 mo) & $1(4.4)$ & $0(0.0)$ & $*$ \\
\hline New (3 mo-1 yr) & $4(17.4)$ & $3(27.3)$ & $*$ \\
\hline Experienced (1-5 yr) & $3(13.0)$ & 5 (45.5) & $*$ \\
\hline Very Experienced ( $\geq 5$ yr) & $15(65.2)$ & $3(27.3)$ & $*$ \\
\hline
\end{tabular}

with the existing prosthesis and equivalent in the five remaining dexterity tests. Spontaneity of use, as measured by the UNB, was significantly better with the DEKA Arm than with the existing device. Self-reported activity performance (i.e., the number of daily activities performed using the prosthesis and difficulty with patient-specific activities) was significantly better with the DEKA Arm than with the existing prosthesis. Subjects reported (on the UEFS use scale) that they performed significantly more activities with a prosthesis when using the DEKA Arm than they did using their existing prosthesis. Subjects also reported less difficulty with activities that were important to them (listed on the PSFS) when using the DEKA Arm than with their existing prosthesis. Since results of Wilcoxon signed-ranks tests were similar, these results are not shown.

The results of Wilcoxon signed-ranks tests show that score differences in dexterity scores for existing prosthe- sis and the DEKA Arm varied by prosthetic fit level (Table 5). Subjects using the RC prosthesis had worse BB scores and JTHF feeding and light cans scores when using the DEKA Arm than with their existing prosthesis and equivalent scores on four other dexterity tests. Subjects using HC prostheses had lower BB scores, better JTHF heavy and light can scores, and equivalent scores on four other tests. However, subjects using the SC had better dexterity as measured by JTHF page turning but equivalent scores on the seven other dexterity tests.

Analyses show that spontaneity of prosthetic use and activity performance was better for users of the DEKA Arm at the SC level but equivalent at the RC and HC levels. In contrast, self-reported prosthetic use and difficulty performing patient-specific activities were superior with the DEKA Arm than with the existing prosthesis for all levels of users. 


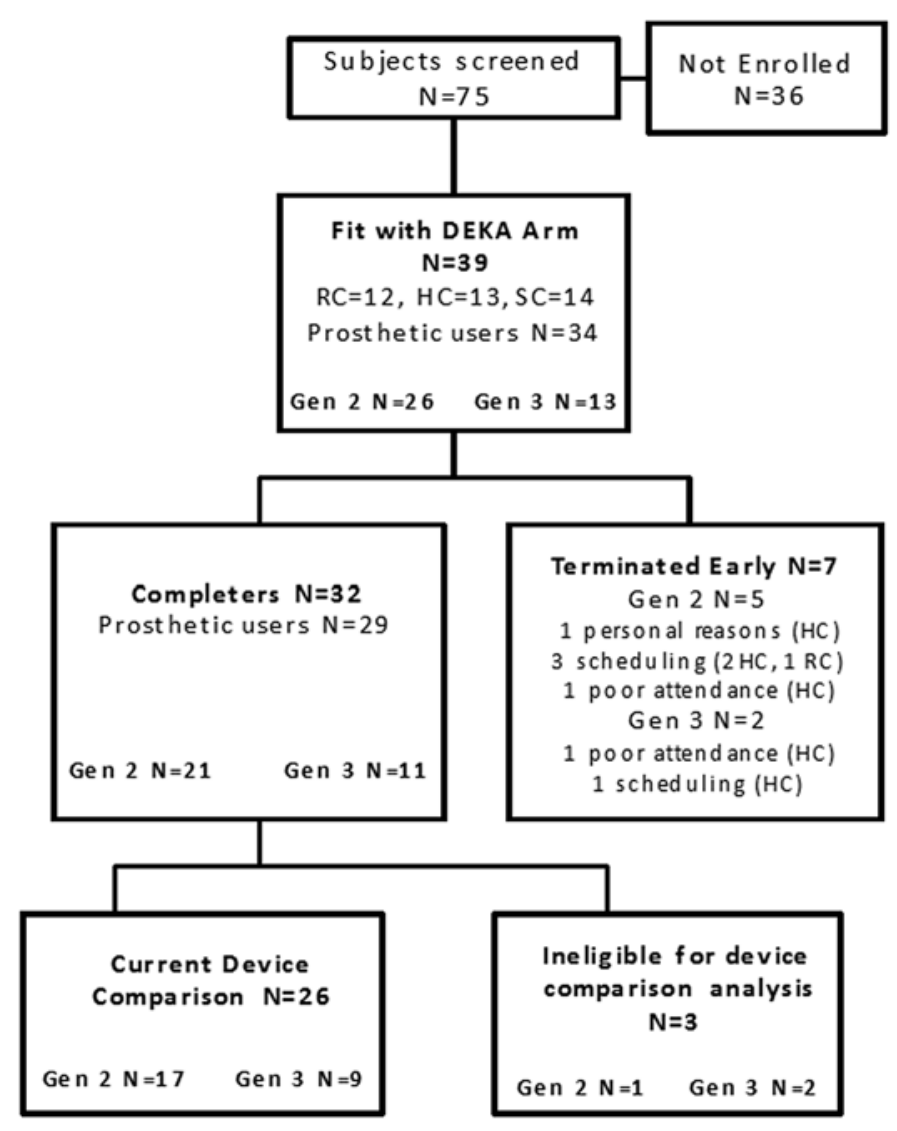

Figure.

Flow diagram of subjects in study. gen $2=$ second-generation DEKA Arm, gen 3 = third-generation DEKA Arm, $\mathrm{HC}$ = humeral configuration, $\mathrm{RC}=$ radial configuration, $\mathrm{SC}=$ shoulder configuration.

\section{DISCUSSION}

This is the first study to report on dexterity, activity performance, and prosthetic skill and spontaneity achieved by VA subjects after training to use the DEKA Arm. Overall, we found that RC users had the best dexterity, followed by HC users and then SC users. A slightly different pattern was observed in activity performance, with RC and HC users having comparable scores and SC users demonstrating worse performance. Interestingly, no differences in self-reported functional performance were observed by configuration level, suggesting that dexterity was not a primary factor in perceived difficulty of activity performance.

Our study is the first to compare outcomes of subjects using a DEKA Arm and their own prosthetic device. Subjects at all amputation levels reported that they could per- form more activities using the DEKA Arm (as measured by the UEFS) than they could perform with their existing prosthesis and that they had less difficulty performing self-selected activities (as measured by the PSFS) when using the DEKA Arm. As a group, dexterity was equivalent or somewhat diminished with the DEKA Arm than with the existing prosthesis, although the effect differed by configuration level. RC users had significantly worse dexterity on three of eight tests, although the mean scores were lower for all dexterity tests. HC users had mixed dexterity scores (2 of 8 tests were significantly better and 1 of 8 tests was significantly worse), and SC users had equivalent or somewhat improved dexterity ( 1 of 8 tests was better). The participants with upper-limb amputation in our study had substantially impaired BB scores compared with established norms. Published normative values for 45 to $49 \mathrm{yr}$ old males show that persons of this age transport 75.8 to 76.9 blocks in $1 \mathrm{~min}$ [19]. We found that DEKA Arm users transported an average of 5.1 (SC users) to 14.1 (RC users) while current prosthesis users transported an average of 4.5 (SC users) to 25.6 (RC users) blocks in $1 \mathrm{~min}$.

Our results triangulate well with the findings from our qualitative analyses [17,33]. DEKA Arm users at all levels thought that the DEKA Arm was beneficial, with the majority of users reporting that they could perform activities using the DEKA Arm that they could not with their existing prostheses [17]. Our data suggest that perceived functional benefits, particularly in the area of self-care activities, were somewhat greater for SC users [17]. The findings from the current study suggest that the benefits of increased independence and less difficulty in performance of functional activities may be more highly valued by participants with amputation than speed of fine motor activities (dexterity).

\section{STUDY LIMITATIONS}

Our results need to be interpreted cautiously because we have no way of knowing for certain whether or not the training received in this protocol was sufficient to maximize each and every subject's performance. All subjects participated in a semistructured, in-laboratory training program, where the lengths of visits, as well as the number of visits, were specified by the study protocol. Training was considered complete when the prespecified number of visits had been achieved. In most cases, training intensity was 
Table 2.

Performance-based and self-report measure scores (mean \pm standard deviation [range]) at end of study by DEKA Arm configuration level (second-generation and third-generation DEKA Arms).

\begin{tabular}{|c|c|c|c|c|c|}
\hline $\begin{array}{c}\text { Performance-Based } \\
\text { Measure }\end{array}$ & RC & HC & SC & $p$-Value & All \\
\hline No. of Subjects & 11 & 7 & 14 & & 32 \\
\hline $\mathrm{BB}$ & $13.4 \pm 5.7(6-21)$ & $9.1 \pm 2.6(6-13)$ & $4.5 \pm 3.7(0-11)$ & $<0.01$ & $8.6 \pm 5.8(0-21)$ \\
\hline \multicolumn{6}{|l|}{ JTHF $^{*}$} \\
\hline Writing & $0.45 \pm 0.20(0.23-0.84)$ & $0.37 \pm 0.15(0.13-0.58)$ & $0.25 \pm 0.10(0.07-0.44)$ & $<0.01$ & $0.34 \pm 0.17(0.07-0.84)$ \\
\hline Page Turning & $0.09 \pm 0.04(0.05-0.17)$ & $0.06 \pm 0.03(0.02-0.12)$ & $0.03 \pm 0.02(0.00-0.05)$ & $<0.01$ & $0.06 \pm 0.04(0.00-0.17)$ \\
\hline Small Items & $0.09 \pm 0.06(0.00-0.18)$ & $0.05 \pm 0.03(0.03-0.10)$ & $0.03 \pm 0.02(0.00-0.07)$ & $<0.01$ & $0.06 \pm 0.05(0.00-0.18)$ \\
\hline Feeding & $0.12 \pm 0.07(0.00-0.27)$ & $0.08 \pm 0.03(0.03-0.13)$ & $0.03 \pm 0.02(0.00-0.06)$ & $<0.01$ & $0.07 \pm 0.06(0.00-0.27)$ \\
\hline Light Cans & $0.23 \pm 0.10(0.10-0.38)$ & $0.17 \pm 0.06(0.09-0.24)$ & $0.09 \pm 0.06(0.03-0.20)$ & $<0.01$ & $0.16 \pm 0.10(0.03-0.38)$ \\
\hline Heavy Cans & $0.23 \pm 0.10(0.09-0.39)$ & $0.19 \pm 0.06(0.11-0.27)$ & $0.11 \pm 0.06(0.05-0.21)$ & $<0.01$ & $0.17 \pm 0.09(0.05-0.39)$ \\
\hline No. of Subjects & 9 & 7 & 11 & & 27 \\
\hline \multicolumn{6}{|l|}{ UNB } \\
\hline Skill & $2.9 \pm 0.5(2.3-3.7)$ & $3.3 \pm 0.1(3.2-3.4)$ & $3.0 \pm 0.4(2.4-3.8)$ & 0.17 & $3.0 \pm 0.4(2.3-3.8)$ \\
\hline Spontaneity & $3.1 \pm 0.5(2.4-3.9)$ & $3.5 \pm 0.2(3.2-3.7)$ & $3.2 \pm 0.4(2.5-3.8)$ & 0.08 & $3.2 \pm 0.4(2.4-3.9)$ \\
\hline No. of Subjects & 10 & 7 & 11 & & 28 \\
\hline AM-ULA & $1.9 \pm 0.4(1.4-2.4)$ & $1.9 \pm 0.3(1.5-2.3)$ & $1.4 \pm 0.3(1.1-1.9)$ & 0.01 & $1.7 \pm 0.4(1.1-2.4)$ \\
\hline Self-Report Measure & RC & HC & SC & $p$-Value & All \\
\hline No. of Subjects & 11 & 7 & 14 & & 32 \\
\hline UEFS & $42.0 \pm 5.8(30.8-49.0)$ & $38.7 \pm 10.5(22.7-52.3)$ & $43.3 \pm 5.8(30.8-50.7)$ & 0.60 & $41.8 \pm 7.0(22.7-52.3)$ \\
\hline UEFS Use & $1.0 \pm 0.0(0.9-1.0)$ & $0.9 \pm 0.2(0.5-1.0)$ & $0.7 \pm 0.4(0.0-1.0)$ & 0.13 & $0.8 \pm 0.3(0.0-1.0)$ \\
\hline PSFS & $5.7 \pm 2.1(1.7-8.8)$ & $6.0 \pm 1.3(3.5-7.8)$ & $5.3 \pm 2.0(2.0-8.7)$ & 0.59 & $5.6 \pm 1.9(1.7-8.8)$ \\
\hline
\end{tabular}

${ }^{*}$ Number of items per second.

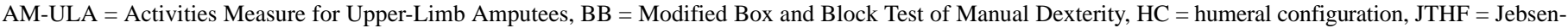

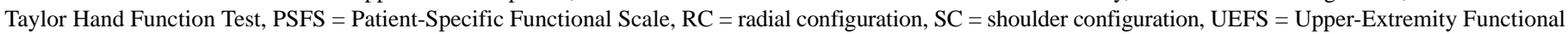
Scale, UNB = University of New Brunswick Test of Prosthetic Function for Unilateral Amputees.

Table 3.

Comparison of scores (mean \pm standard deviation) by DEKA Arm prototype by configuration level.

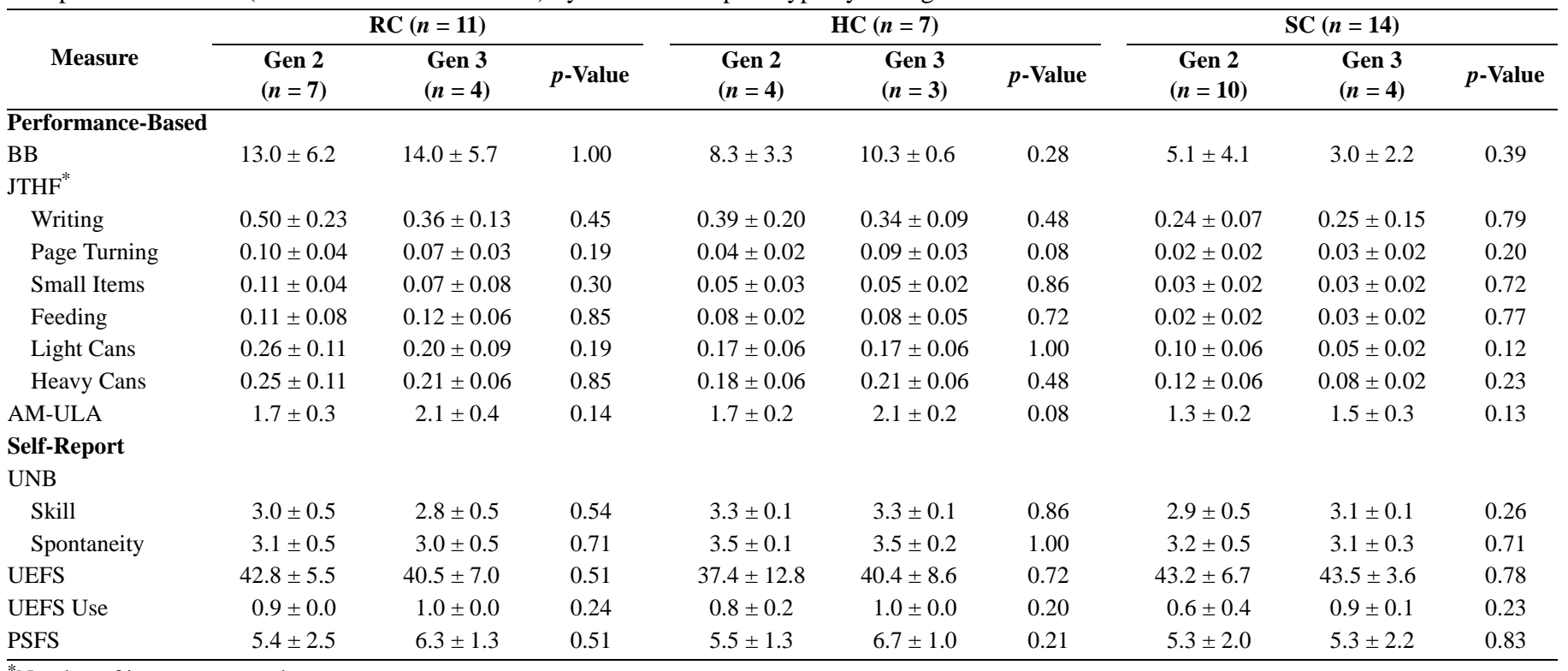

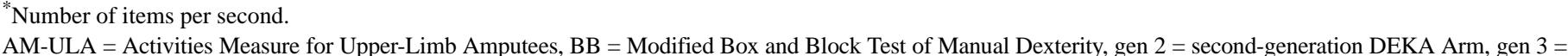

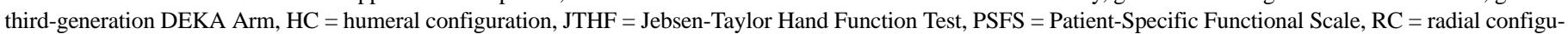

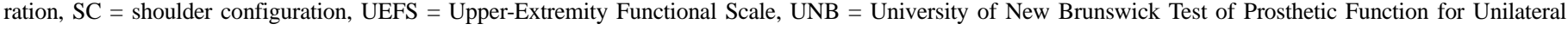
Amputees. 
Table 4.

Outcome scores (mean \pm standard deviation [range]) using existing prosthesis compared with DEKA Arm.

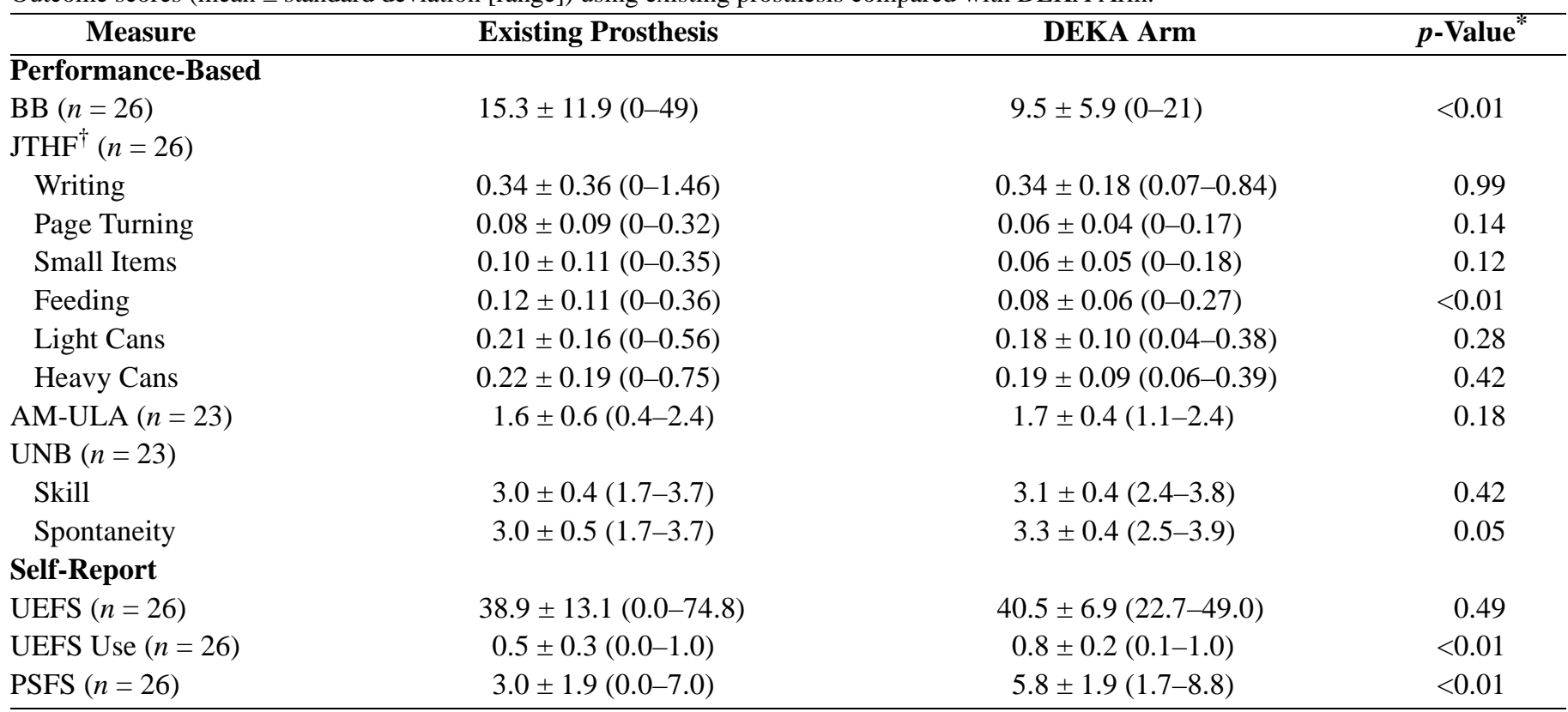

${ }^{*}$ Paired $t$-test.

${ }^{\dagger}$ Number of items per second.

AM-ULA = Activities Measure for Upper-Limb Amputees, BB = Modified Box and Block Test of Manual Dexterity, JTHF = Jebsen-Taylor Hand Function Test, PSFS = Patient-Specific Functional Scale, UEFS = Upper-Extremity Functional Scale, UNB = University of New Brunswick Test of Prosthetic Function for Unilateral Amputees.

Table 5.

Comparison scores (mean) of existing prosthesis with DEKA Arm by configuration level $(n=26)$.

\begin{tabular}{|c|c|c|c|c|c|c|c|c|c|}
\hline \multirow[b]{2}{*}{ Measure } & \multicolumn{3}{|c|}{$\operatorname{RC}(n=10)$} & \multicolumn{3}{|c|}{ HC $(n=6)$} & \multicolumn{3}{|c|}{$\mathrm{SC}(n=10)$} \\
\hline & $\begin{array}{c}\text { Existing } \\
\text { Prosthesis }\end{array}$ & DEKA Arm & $p$-Value & $\begin{array}{c}\text { Existing } \\
\text { Prosthesis }\end{array}$ & DEKA Arm & $p$-Value & $\begin{array}{c}\text { Existing } \\
\text { Prosthesis }\end{array}$ & DEKA Arm & $p$-Value \\
\hline \multicolumn{10}{|c|}{ Performance-Based } \\
\hline $\mathrm{BB}$ & 25.6 & 14.1 & 0.02 & 16.33 & 9.33 & 0.03 & 4.5 & 5.1 & 0.72 \\
\hline \multicolumn{10}{|l|}{$\mathrm{JTHF}^{*}$} \\
\hline Writing & 0.57 & 0.44 & 0.58 & 0.26 & 0.33 & 0.25 & 0.16 & 0.23 & 0.07 \\
\hline Feeding & 0.21 & 0.12 & 0.01 & 0.11 & 0.08 & 0.35 & 0.04 & 0.03 & 0.30 \\
\hline Light Cans & 0.38 & 0.24 & 0.03 & 0.13 & 0.18 & 0.03 & 0.08 & 0.11 & 0.39 \\
\hline Heavy Cans & 0.39 & 0.24 & 0.06 & 0.14 & 0.21 & 0.05 & 0.10 & 0.13 & 0.72 \\
\hline AM-ULA & 2.1 & 2.0 & 0.33 & 1.8 & 1.9 & 0.60 & 0.9 & 1.3 & 0.03 \\
\hline \multicolumn{10}{|l|}{ UNB } \\
\hline UEFS Use & 0.5 & 1.0 & $<0.01$ & 0.2 & 0.9 & 0.03 & 0.5 & 0.8 & 0.03 \\
\hline PSFS & 4.1 & 5.7 & 0.04 & 2.9 & 6.4 & 0.03 & 1.8 & 5.7 & 0.01 \\
\hline
\end{tabular}

*Number of items per second.

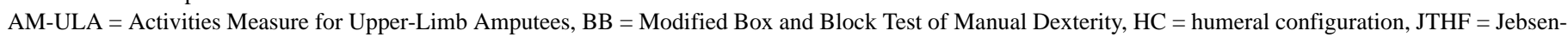

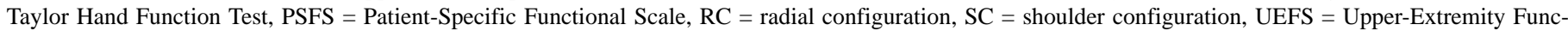
tional Scale, UNB = University of New Brunswick Test of Prosthetic Function for Unilateral Amputees. 
3 to 5 days per week and sessions lasted $2 \mathrm{~h}$ and included additional time for rest breaks. Several subjects attended less often because of scheduling problems, illness, or need for device repairs. We recognize that the amount of training or the schedule of training provided in our study may have been insufficient for some subjects and more than enough for others. Clinical experience suggests that training time needs to be longer or more intensive for older persons and those with cognitive impairments [34]. Based on early feedback from subjects and clinical staff, we determined that $20 \mathrm{~h}$ of training was not sufficient for SC users, and we added $10 \mathrm{~h}$ of additional training. We have no way of knowing whether or not subjects would have continued to gain proficiency if given additional time and training.

There are limitations to the comparisons of existing devices with the DEKA Arm that were conducted in this study. Prosthetic users in our study had received varying amounts of prior prosthetic training, had widely differing years of prosthetic experience, and used all types of devices. In some cases, subjects had decades of prior prosthetic experience and in others, only months or years. We attempted to ameliorate differences in prosthetic experience (or at least bias comparisons toward the null) by excluding a very new prosthetic user from this comparison. All of our subjects had less than $30 \mathrm{~h}$ of training in use of the DEKA Arm, which was delivered over weeks to several months. Thus, all subjects had been using their existing prostheses for far longer than they had used the DEKA Arm.

Subjects in our study had all types of devices: some myoelectric, some body-powered, and others hybrid. A variety of terminal devices were used, including standard hooks and various myoelectric hands. We did not compare outcomes using the DEKA Arm with outcomes for subtypes of current prostheses. It is possible that comparisons for subtypes of existing devices would yield somewhat difference results. However, we do not believe that the differences would be profound because of the key features of the DEKA Arm that were not available in any existing prostheses. All levels of DEKA Arm have powered wrist flexion and extension and six grips, and all subjects controlled the DEKA Arm movement, in part or in full, with foot controls that are not available in any existing prostheses. The HC and SC DEKA Arms have powered humeral rotation. The SC DEKA Arm has a powered shoulder and uses an end-point control scheme. Future studies could compare the DEKA Arm with specific types of existing prostheses.
For pragmatic reasons, we made no attempt to alter the subject's prosthetic prescription to standardize the type of comparison device used in the study. Thus, it is likely that some subjects were tested with an existing prosthesis that was not optimal for their needs. It is conceivable that, given the latest available technology and sufficient training, some might have performed better than they did on baseline testing with their existing prostheses. However, we have no way of testing this hypothesis without a different study design. Such a design was not feasible in our optimization study due to challenges in recruiting a scarce population and the extensive time and costs involved in prescribing standardized devices and then training prosthetic users prior to baseline testing.

Lastly, there are also limitations to the generalizability of our findings to the overall population with upper-limb amputation. Our sample was purposefully chosen to provide a diverse range of users at each of three device configuration levels and to include participants with bilateral and unilateral amputation as well as of both sexes. Although we have no data on population characteristics, we believe that our subject pool represented the diversity of the population with upper-limb amputation but was not representative of the U.S. or Veteran population.

\section{CONCLUSIONS}

This study reported on dexterity, activity performance, prosthetic skill and spontaneity, and self-reported function after training with the DEKA Arm. Although dexterity varied by amputation level, self-reported function and number of activities performed using the prosthesis were similar across amputation levels. Comparisons of the DEKA Arm with existing prostheses showed that the effect of the DEKA Arm on dexterity was equivalent or mixed. Activity performance and spontaneity of prosthetic use improved for SC users, whereas use of the prosthesis to perform activities and perceived difficulty in performing self-selected tasks improved for participants of all levels of amputation using the DEKA Arm.

\section{ACKNOWLEDGMENTS}

\section{Author Contributions:}

Principal investigators: L. Resnik, N. Sasson, G. Latlief, L. Smurr-Walters.

Study design: L. Resnik, N. Sasson, G. Latlief, L. Smurr-Walters. 
Study coordination: L. Resnik.

Management of data: M. Borgia.

Analysis of data: L. Resnik.

Statistical analysis: M. Borgia.

Drafting of manuscript: L. Resnik, M. Borgia, N. Sasson, G. Latlief, L. Smurr-Walters.

Obtained funding: L. Resnik.

Financial Disclosures: The authors have declared that no competing interests exist.

Funding/Support: This material was based on work supported by the VA RR\&D Service (grants A6780 and A6780I). DEKA's support of the VA Optimization Study was sponsored by DARPA and the U.S. Army Research Office.

Additional Contributions: The authors acknowledge the valuable work of study coordinator Kate Barnabe and members of the research teams at all study sites. As of the publication date of this article, the DEKA Arm system is currently being reviewed by the U.S. Food and Drug Administration and has not received clearance to be commercially marketed.

Institutional Review: This research was approved by the institutional review boards of the Providence VA Medical Center, VA NYHHS, James A. Haley Veterans' Hospital, VA Long Beach Healthcare System, and Center for the Intrepid.

Participant Follow-Up: The authors do not plan to inform participants of the publication of this study.

Disclaimer: The information in this article does not necessary reflect the position or policy of the U.S. Government; no official endorsement should be inferred. The view(s) expressed herein are those of the author(s) and do not reflect the official policy or position of BAMC, U.S. Army Medical Department, U.S. Army Office of the Surgeon General, Department of the Army, Department of Defense, or U.S. Government. Additionally, the appearance of name-brand products in this article does not constitute endorsement of the information, products, or services contained therein by BAMC, U.S. Army Medical Department, U.S. Army Office of the Surgeon General, Department of the Army, Department of Defense, or U.S. Government.

\section{REFERENCES}

1. Biddiss EA, Chau TT. Upper limb prosthesis use and abandonment: A survey of the last 25 years. Prosthet Orthot Int. 2007;31(3):236-57. [PMID:17979010]

2. Wright TW, Hagen AD, Wood MB. Prosthetic usage in major upper extremity amputations. J Hand Surg Am. 1995; 20(4):619-22. [PMID:7594289]

3. Biddiss E, Beaton D, Chau T. Consumer design priorities for upper limb prosthetics. Disabil Rehabil Assist Technol. 2007;2(6):346-57. [PMID:19263565] http://dx.doi.org/10.1080/17483100701714733

4. Atkins DJ, Heard DC, Donovan WH. Epidemiologic overview of individuals with upper-limb loss and their reported research priorities. J Prosthet Orthot. 1996;8(1):2-11.

5. Dakpa R, Heger H. Prosthetic management and training of adult upper limb amputees. Curr Orthop.1997;11(3):193-202.
6. Reyburn TV. A method of early prosthetics training for upper-extremity amputees. Artif Limbs. 1971;15(2):1-5. [PMID:5135222]

7. Bhaskaranand K, Bhat AK, Acharya KN. Prosthetic rehabilitation in traumatic upper limb amputees (an Indian perspective). Arch Orthop Trauma Surg. 2003;123(7):363-66. [PMID:12827395] http://dx.doi.org/10.1007/s00402-003-0546-4

8. Maguire MT. Empowering prosthetics. Rehab Manag. 2008; 21(10):10. [PMID:19263992]

9. Lake C, Dodson R. Progressive upper limb prosthetics. Phys Med Rehabil Clin N Am. 2006;17(1):49-72. [PMID:16517345] http://dx.doi.org/10.1016/j.pmr.2005.10.004

10. Biddiss EA, Chau TT. Multivariate prediction of upper limb prosthesis acceptance or rejection. Disabil Rehabil Assist Technol. 2008;3(4):181-92. [PMID:19238719] http://dx.doi.org/10.1080/17483100701869826

11. Biddiss E, Chau T. The roles of predisposing characteristics, established need, and enabling resources on upper extremity prosthesis use and abandonment. Disabil Rehabil Assist Technol. 2007;2(2):71-84. [PMID:19263542] http://dx.doi.org/10.1080/17483100601138959

12. Resnik L, Klinger SL, Etter K. The DEKA Arm: Its features, functionality, and evolution during the Veterans Affairs Study to optimize the DEKA Arm. Prosthet Orthot Int. 2013 Oct 22. Epub ahead of print. [PMID:24150930]

13. Defense Sciences Office [Internet]. Revolutionizing prosthetics. Arlington (VA): Defense Advanced Research Projects Agency; 2014 [cited 2012 Dec 21]. Available from: http://www.darpa.mil/our work/dso/programs/ revolutionizing_prosthetics.aspx

14. Resnik L, Etter K, Klinger SL, Kambe C. Using virtual reality environment to facilitate training with advanced upperlimb prosthesis. J Rehabil Res Dev. 2011;48(6):707-18. [PMID:21938657] http://dx.doi.org/10.1682/JRRD.2010.07.0127

15. Phillips SL, Resnik L, Fantini C, Latlief G. Endpoint control for a powered shoulder prosthesis. J Prosthet Orthot. 2013;25(4):193-200. http://dx.doi.org/10.1097/JPO.0000000000000006

16. Barnum CM. Usability testing and research. New York (NY): Longman; 2002.

17. Resnik L, Latlief G, Klinger SL, Sasson N, Walters LS. Do users want to receive a DEKA Arm and why? Overall findings from the Veterans Affairs Study to optimize the DEKA Arm. Prosthet Orthot Int. 2013 Nov 28. Epub ahead of print. [PMID:24286806]

18. Desrosiers J, Bravo G, Hébert R, Dutil E, Mercier L. Validation of the Box and Block Test as a measure of dexterity of elderly people: reliability, validity, and norms studies. 
Arch Phys Med Rehabil. 1994;75(7):751-55.

[PMID:8024419]

19. Mathiowetz V, Volland G, Kashman N, Weber K. Adult norms for the Box and Block Test of manual dexterity. Am J Occup Ther. 1985;39(6):386-91. [PMID:3160243] http://dx.doi.org/10.5014/ajot.39.6.386

20. Platz T, Pinkowski C, van Wijck F, Kim IH, di Bella P, Johnson G. Reliability and validity of arm function assessment with standardized guidelines for the Fugl-Meyer Test, Action Research Arm Test and Box and Block Test: A multicentre study. Clin Rehabil. 2005;19(4):404-11. [PMID:15929509] http://dx.doi.org/10.1191/0269215505cr832oa

21. Rider B. Comparison of standardized and non-standardized administration of the Jebsen Hand Function Test. J Hand Ther. 1988;1(3):121-23.

22. Resnik L, Adams L, Borgia M, Delikat J, Disla R, Ebner C, Walters LS. Development and evaluation of the activities measure for upper limb amputees. Arch Phys Med Rehabil. 2013;94(3):488-94. [PMID:23085376] http://dx.doi.org/10.1016/j.apmr.2012.10.004

23. Sanderson ER, Scott RN. UNB Test of Prosthetics Function: A test for unilateral upper extremity amputees, ages 2-13. Fredericton (Canada): Institute of Biomedical Engineering, University of New Brunswick; 1985.

24. Burger H, Franchignoni F, Heinemann AW, Kotnik S, Giordano A. Validation of the orthotics and prosthetics user survey upper extremity functional status module in people with unilateral upper limb amputation. J Rehabil Med. 2008;40(5):393-99. [PMID:18461266] http://dx.doi.org/10.2340/16501977-0183

25. Heinemann AW, Bode RK, O’Reilly C. Development and measurement properties of the Orthotics and Prosthetics Users' Survey (OPUS): A comprehensive set of clinical outcome instruments. Prosthet Orthot Int. 2003;27(3):191-206. [PMID:14727700] http://dx.doi.org/10.1080/03093640308726682

26. Stratford P, Gill C, Westaway M, Binkley J. Assessing disability and change on individual patients: A report of a patient specific measure. Physiother Can. 1995;47(4):258-63.

http://dx.doi.org/10.3138/ptc.47.4.258

27. Resnik L, Borgia M. Reliability and validity of outcome measures for upper limb amputation. J Prosthet Orthot. 2012;24(4):192-201. http://dx.doi.org/10.1097/JPO.0b013e31826ff91c

28. Resnik L, Baxter K, Borgia M, Mathewson K. Is the UNB test reliable and valid for use with adults with upper limb amputation? J Hand Ther. 2013;26(4):353-59, quiz 359. [PMID:24012168]

http://dx.doi.org/10.1016/j.jht.2013.06.004

29. Linacre JM. A user's guide to WINSTEPS. Chicago (IL): MESA Press; 1999.

30. Hefford C, Abbott JH, Arnold R, Baxter GD. The patientspecific functional scale: validity, reliability, and responsiveness in patients with upper extremity musculoskeletal problems. J Orthop Sports Phys Ther. 2012;42(2):56-65.

[PMID:22333510]

http://dx.doi.org/10.2519/jospt.2012.3953

31. Chatman AB, Hyams SP, Neel JM, Binkley JM, Stratford PW, Schomberg A, Stabler M. The Patient-Specific Functional Scale: Measurement properties in patients with knee dysfunction. Phys Ther. 1997;77(8):820-29.

[PMID:9256870]

32. Westaway MD, Stratford PW, Binkley JM. The patientspecific functional scale: Validation of its use in persons with neck dysfunction. J Orthop Sports Phys Ther. 1998; 27(5):331-38. [PMID:9580892] http://dx.doi.org/10.2519/jospt.1998.27.5.331

33. Resnik L, Lieberman-Klinger S, Etter K. User and Clinician Perspectives on DEKA Arm: Results of VA study to optimize DEKA Arm. J Rehabil Res Dev. 2014;51(1):27-38. http://dx.doi.org/10.1682/JRRD.2013.03.0068

34. Resnik L, Meucci MR, Lieberman-Klinger S, Fantini C, Kelty DL, Disla R, Sasson N. Advanced upper limb prosthetic devices: Implications for upper limb prosthetic rehabilitation. Arch Phys Med Rehabil. 2012;93(4):710-17. [PMID:22464092] http://dx.doi.org/10.1016/j.apmr.2011.11.010

Submitted for publication August 14, 2013. Accepted in revised form October 30, 2013.

This article and any supplementary material should be cited as follows:

Resnik L, Borgia M, Latlief G, Sasson N, Smurr-Walters L. Self-reported and performance-based outcomes using DEKA Arm. J Rehabil Res Dev. 2014;51(3):351-62. http://dx.doi.org/10.1682/JRRD.2013.08.0180

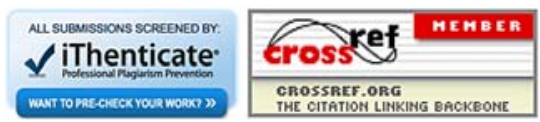


